\title{
Mecanismos para Controle de Tráfego UDP através de Política de Preços Baseada na Utilidade
}

\author{
Rafael D. RIBEIRO, Ronaldo M. SALLES \\ Instituto Militar de Engenharia - IME \\ Seção de Engenharia de Computação - SE/8 \\ Rio de Janeiro, R.J., Brasil. E-mail:\{rdribeiro,salles\}@ime.eb.br
}

\begin{abstract}
Resumo-Este trabalho estuda os problemas decorrentes da coexistência dos fluxos TCP e UDP em redes de dados, tendo em vista o observado aumento do volume de tráfego transportado via UDP nos últimos anos. Nossa proposta é que os fluxos UDP, por não serem cooperativos, sejam penalizados via tarifação de acordo com o impacto causado no tráfego TCP. Algoritmos de controle de admissão e roteamento são propostos de forma a regularem o tráfego UDP minimizando o estrangulamento do TCP e garantindo também a QoS necessária aos fluxos UDP. Resultados da simulação mostram as vantagens da proposta quando comparada com o cenário atual da Internet.
\end{abstract}

Palavras-Chave-qualidade de serviço, coexistência TCP-UDP, controle de congestionamento, controle de admissão, função utilidade

Abstract-This work studies the coexistence of TCP and UDP flows in data networks. Our proposal is that UDP flows are penalized, through a dynamic charging scheme, according to the impact caused on TCP traffic. Admission control and routing algorithms are proposed to regulate UDP traffic minimizing TCP starvation and providing the required QoS for UDP flows. Simulation results show the advantage of our proposal when compared to current procedures in the Internet scenario.

Keywords-quality of service, coexistence of TCP and UDP, congestion control, admission control, utility function

\section{INTRODUÇÃO}

Atualmente a Internet apresenta mais de 800 milhões de usuários e uma taxa de crescimento por volta de $126 \%$ nos últimos seis anos [1]. Com o aumento do número de usuários e a popularização das ofertas de serviço de banda larga residenciais, o número de novas aplicações e seus usuários também vem crescendo.

As aplicações geralmente transportam seus dados pela Internet por meio dos protocolos TCP ou UDP. O TCP é um protocolo reativo que responde ao sinal de congestionamento reduzindo sua janela de transmissão, e consequentemente a taxa com que os dados são injetados na rede. O objetivo desse procedimnto é cooperar com o reestabelecimento da condição normal de operação da rede. Além disso, o TCP garante a ordenação dos pacotes no destino e, em caso de falha na transmissão, realiza a retransmissão da informação perdida. Por outro lado, o UDP não prevê técnicas de confirmação de entrega nem realiza qualquer tipo de controle de congestionamento. Seu objetivo é transmitir dados com o maior desempenho possível. Assim, fica a cargo da aplicação escolher o protocolo de transporte mais adequado às características do seu fluxo de dados.
Aplicações que geram fluxos sensíveis a perdas e pouco sensíveis ao atraso, os chamados fluxos elásticos [4] como por exemplo o SMTP (Simple Mail Transfer Protocol), geralmente utilizam o protocolo TCP. Já aplicações menos sensíveis a perdas, porém sensíveis ao atraso, por exemplo as aplicações multimídia e de tempo-real como Voz sobre IP (VoIP), tendem a utilizar o protocolo UDP. Desta forma, os recursos de rede são compartilhados por ambos os fluxos TCP e UDP.

A coexistência dos protocolos UDP e TCP em uma mesma rede é nociva aos fluxos TCP principalmente em situações de congestionamento. Ao sinal de congestionamento, o mecanismo de controle do TCP irá reduzir a sua taxa de transmissão e o UDP irá manter a taxa desejada sem que haja nenhum controle que o regule. O agravamento dessa condição leva os fluxos TCP ao estrangulamento e a uma alocação injusta dos recursos de rede.

O trabalho de Sally e Kevin [5] apresenta um estudo sobre o comportamento dos fluxos TCP e UDP ao competirem por largura de banda em um enlace. Enquanto a taxa dos fluxos UDP é baixa, o número de pacotes TCP entregues é alto. Porém, quando a taxa de envio dos fluxos UDP é maior, o tráfego UDP começa a utilizar uma grande parte do enlace acarretando em um aumento na perda de pacotes TCP. O mecanismo de controle de congestionamento TCP responde então reduzindo sua própria taxa de transmissão de forma a permitir que os fluxos UDP dominem o enlace. Assim podese perceber que os fluxos UDP "estrangulam" os fluxos TCP, reduzindo sua taxa de transmissão quase a zero.

Atualmente os fluxos TCP são responsáveis pelo transporte de $90 \%$ do tráfego circulante na internet; o restante é transportado pelo UDP [8]. Com o crescimento do número de usuários e aplicações que utilizam o UDP como protocolo de transporte, existe uma grande probabilidade de que estes valores se alterem aumentando cada vez mais o percentual dos fluxos UDP. Caso não haja um mecanismo para regular estes fluxos, poderá ocorrer um estrangulamento do tráfego TCP.

Este artigo apresenta um mecanismo capaz de controlar os fluxos UDP e permitir uma coexistência mais harmônica com o tráfego TCP. Utilizamos conceitos da teoria microeconômica no desenvolvimento de uma proposta de algoritmo de roteamento e de controle de admissão para preservar os fluxos TCP e garantir qualidade de serviço (QoS) aos fluxos UDP em enlaces congestionados.

Este trabalho está organizado da seguinte forma. Na seção 
2, são abordados os conceitos de funções utilidade da teoria microeconômica e a forma como eles se aplicam na modelagem do comportamento dos fluxos TCP e UDP. Na seção 3, apresentamos o mecanismo de preço dinâmico que será utilizado pelos algoritmos propostos. Na seção 4 , os algoritmos de controle de admissão e roteamento são apresentados. A seção 5 apresenta os cenários de simulação e os resultados obtidos. O trabalho é concluido na seção 6 .

\section{FunÇÕes Utilidade E PReÇO}

Função Utilidade é um conceito da microeconomia que representa a preferência, satisfação ou prazer de um consumidor por uma determinada cesta de consumo em relação a outra, isto é, a preferência de um consumidor optar (pagar a mais, esperar a mais, ficar mais feliz com, etc) por um determinado produto a outro [3]. Para o nosso estudo o conceito de função utilidade está relacionado com a satisfação do usuário (ou aplicação) em utilizar/consumir determinado recurso (capacidade de transmissão dos enlaces, largura de banda em bps) disponível na rede. As funções utilidades para os tipos de tráfego UDP e TCP serão definidas nos próximos tópicos.

\section{A. Função Utilidade para Tráfego TCP $\left(U_{t c p}\right)$}

Baseado no princípio que a utilidade é a forma de medir o grau de satisfação do usuário em relação ao consumo ou ao serviço prestado, a utilidade para aplicações que geram tráfego TCP está relacionada com o desempenho da rede ao transportar este tráfego. Assim, podemos considerar que a utilidade seria medida pela relação entre os pacotes enviados úteis (Goodput) e todos os pacotes enviados, inclusive os retransmitidos (Throughput).

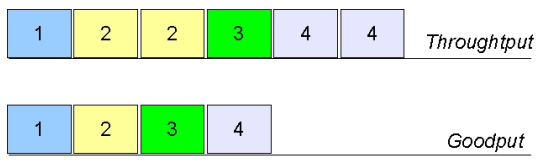

Fig. 1. Representação do Throughput e do Goodput

Considerando $x$ como o throughput e $p$ a probabilidade de perda de pacote, o goodput é dado por $(1-p) x$. Assim: $U_{t c p}=$ goodput/throughput $=(1-p) x / x=1-p$

Segundo os trabalhos [6][7], a probabilidade de perda pode ser definida em função do crescimento da janela de congestionamento TCP para perdas periódicas por:

$$
p=\left(\frac{M S S}{R T T}\right)^{2} \frac{1}{x^{2}}
$$

onde, MSS = Maximum Segment Size e RTT = Round Trip Time.

Determinando $B_{\min }$ como uma constante mínima para a taxa de transmissão dos fluxos TCP, equivalente à transmissão de um único segmento por RTT (janela de tamanho unitário), temos $B_{\min }=M S S / R T T$. Assim, a função utilidade para fluxos TCP $\left(U_{t c p}\right)$ é dada pela equação abaixo e ilustrada na Fig.2.

$$
U_{t c p}=1-\left(\frac{B_{m i n}}{x}\right)^{2}
$$

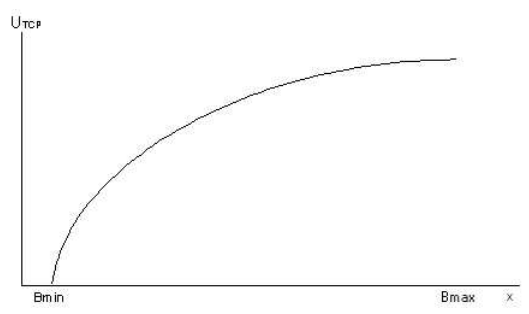

Fig. 2. Curva da função utilidade (Eq. 2) para fluxos TCP

\section{B. Função Utilidade para Tráfego UDP $\left(U_{u d p}\right)$}

Uma aplicação de transmissão de audio/vídeo gera amostras em intervalos regulares. $\mathrm{O}$ tamanho da amostra (quantidade de

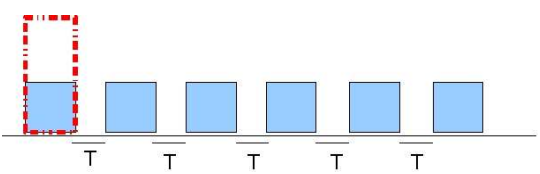

Fig. 3. Amostras de audio/vídeo geradas em intervalos regulares $\mathrm{T}$

bits) está relacionada com a qualidade da informação transmitida. Então pode-se considerar que quanto maior a capacidade de transmissão disponível para os fluxos UDP maior será a utilidade de suas aplicações. Porém, caso a rede não ofereça uma disponibilidade mínima $\left(B_{\min }\right)$, não interessa reservar determinado recurso já que este não atenderia os requisitos mínimos necessários para a aplicação UDP (utilidade zero). No outro extremo, a partir de determinada disponibilidade $\left(B_{\max }\right)$, mais recursos não aumentariam o grau de satisfação dos usuários com o desempenho das aplicações, acarretando em um desperdício de banda.

Assim consideramos $B_{\min }$ como a taxa mínima de transmissão para uma aplicação multimídia, isto é, o pior nível de QoS aceitável e $B_{\max }$ a melhor situação possível. Assumindo uma variação linear de qualidade entre $B_{\min }$ e $B_{\max }$, temos a $U_{u d p}$ definida por:

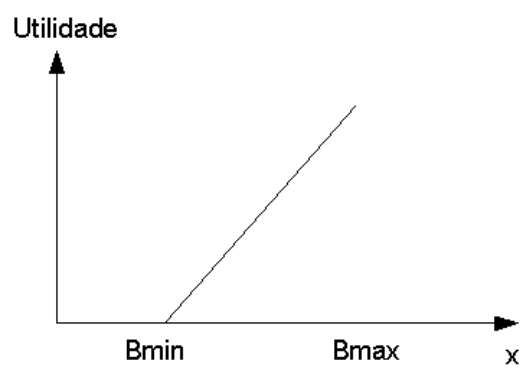

Fig. 4. Função Utilidade UDP por largura de banda (bps) disponível 


$$
U_{u d p}=\left\{\begin{array}{lc}
0, & x \leq B_{\min } \\
1, & x \geq B_{\max } \\
\frac{x-B_{\min }}{B_{\max }-B_{\min }}, & B_{\min }<x<B_{\max }
\end{array}\right.
$$

Onde: $x$ é a banda disponível para o tráfego da aplicação UDP

\section{Política de Preços}

Para tentar prevenir ou minimizar o impacto sofrido pelos fluxos TCP coexistindo com fluxos UDP em redes congestionadas, devemos criar mecanismos capazes de controlar a admissão de novos fluxos UDP e tentar encaminhá-los por rotas onde a rede apresente o menor grau de congestionamento. Assim tenta-se minimizar o impacto de sua transmissão e permitir que os fluxos UDP coexistam de forma mais justa com as conexões TCP. Para isso desenvolvemos mecanismos de preços baseados no princípo da utilidade marginal, no nosso trabalho introduzimos o termo "desutilidade marginal" que será apresentado abaixo.

\section{A. "Desutilidade Marginal"}

Em termos econômicos, a utilidade marginal representa a utilidade adicional com o ganho de uma unidade de um produto $x$ ou, mais formalmente, a derivação da utilidade em relação a $x$ :

$$
U_{\text {marginal }}=\frac{d U}{d x}
$$

A utilidade marginal está associada ao preço que o consumidor está disposto a pagar por unidade adicional do produto $x$ [3].

O conceito de "desutilidade marginal" poderia ser entendido então como a perda que o consumidor experimenta por unidade do produto $x$ que deixa de consumir, ou seja, o grau de insatisfação por deixar de consumir uma unidade de $x$.

O objetivo é fazer com que o UDP pague pela insatisfação causada ao TCP e desta forma, criar um mecanismo automático de regulação do UDP através de política de preços.

Como exemplo imaginemos uma situação onde os fluxos TCP gozam de bom desempenho (rede descongestionada). Uma pequena redução na transmissão destes fluxos não iria gerar um alto grau de insatisfação visto que o TCP é adaptativo (função utilidade é côncava). Neste caso, o fluxo UDP poderia ser acomodado sem maiores problemas e sendo assim seria tarifado um valor baixo. Entretanto, na situação oposta a entrada do UDP iria causar grande insatisfação ("desutilidade") aos TCP e portanto o UDP deveria ser penalizado por isso através de uma tarifação elevada. Esse mecanismo iria desmotivar os fluxos UDP a ingressarem na rede quando o desempenho TCP não está bom, evitando assim o estrangulamento.

Podemos ilustrar o funcionamento desse mecanismo através da análise da aproximação linear (piecewise linear) da função utilidade TCP representada na figura 5. Na aproximação dividimos o eixo da utilidade em 10 segmentos iguais: $0 \leq$ $U_{t c p}<0.1,0.1 \leq U_{t c p}<0.2, \ldots, 0.9 \leq U_{t c p}<1$. Dentro de cada segmento a desutilidade marginal é constante e igual a:

$$
D=\frac{\Delta U_{t c p}}{\Delta x}=0.1 \Delta x^{-1}
$$

Sendo $D_{k}$ a desutilidade relativa ao segmento $k$, pela concavidade da função utilidade temos que: $D_{1}>D_{2}>\ldots>D_{10}$.

Um valor alto de $D_{k}$ significa baixo desempenho TCP e portanto a entrada de tráfego UDP causaria grande impacto, assim a admissão UDP nessas condições só poderia ocorrer sob tarifação elevada a fim de desmotivar sua entrada na rede. O caso oposto ocorre quando $D_{k}$ é baixo (por exemplo $D_{10}$ ), a admissão UDP seria motivada por baixa tarifação.

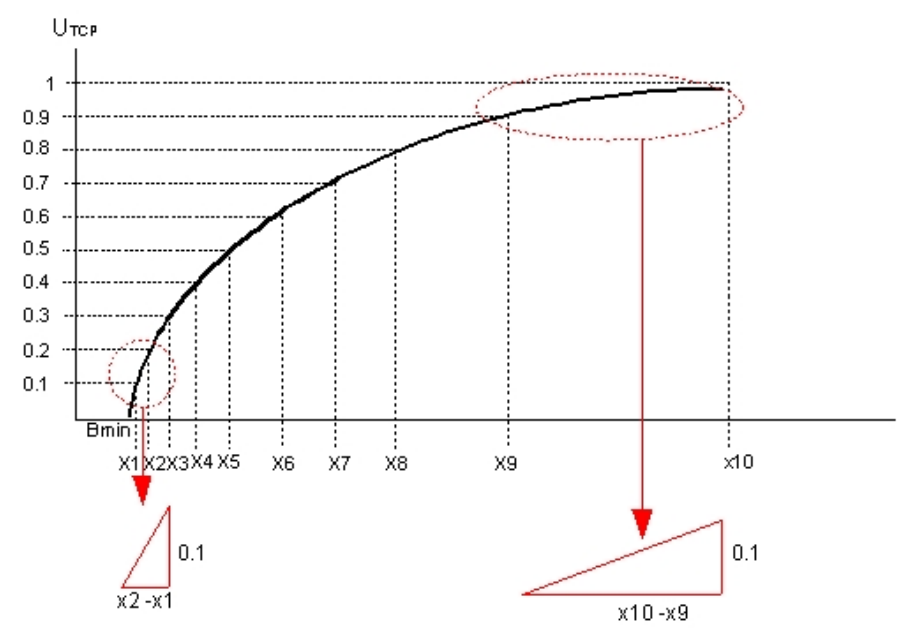

Fig. 5. Representação da inclinação da função $U_{t c p}$ na aproximação piecewise linear

O mecanismo de tarifação regulador deve ser implementado de forma que o preço cobrado por unidade de banda reservada para um fluxo UDP seja proporcional a desutilidade marginal causada nos fluxos TCP por este compartilhamento de banda. Assim, o preço $(\mathrm{P})$ para um tráfego UDP entrar na rede é dado pela expressão:

$$
P=D_{k} \cdot C \cdot Q
$$

Onde:

- $D_{k}$ é a desutilidade marginal do TCP

- C é uma constante monetária

- Q é a quantidade de banda reservada

O mecanismo de preço dinâmico, apresentado nesta seção, irá refletir o nível de congestionamento nos enlaces. Desta forma pode-se tratar a entrada (ou não) de um novo fluxo UDP e a escolha da rota que trará maior utilidade gerando o menor prejuízo para os fluxos TCP existentes na rede.

\section{Algoritmos de Controle de Admiss Ão E ROTEAMENTO}

Como nosso objetivo é regular e controlar os fluxos UDP, que não são sensíveis aos sinais de congestionamento, este trabalho concentra os esforços em criar um novo mecanismo de roteamento e controle de admissão para os fluxos UDP, já que os fluxos TCP já possuem mecanismos de controle sensíveis aos sinais de congestionamento. 


\section{A. Algoritmo de Roteamento}

Para os fluxos UDP a seguinte abordagem foi adotada. Quando um fluxo deseja entrar na rede, ele informa seus parâmetros de $B_{\min }$ e $B_{\max }$, então é acionado o algoritmo Surplus que irá determinar a melhor rota para o novo fluxo. O Surplus é a maximização da diferença entre o caminho que dará a maior utilidade ao fluxo UDP (o qual irá gerar a maior reserva de banda) com o menor preço (já que o caminho de menor preço é o que, caso este novo fluxo seja admitido, trará o menor impacto aos fluxos TCP existentes).

$$
\max \left\{U_{u d p}-\beta \sum_{i=1}^{n} P_{i}\right\}
$$

Onde:

- $P_{i}=$ preço cobrado pela desutilidade TCP no enlace $i$

- $\beta=$ índices de normalização

Observe que $P_{i}$ depende da estimação de $D_{k}$ pelos nós interiores da rede. Esses nós devem propagar a informação $D_{k}$ para os nós de acesso de forma que as decisões de roteamento e admissão possam ser tomadas. O parâmetro $D_{k}$ pode ser estimado através de monitoramento de um ou mais fluxos TCP que usam o link $i$, para este trabalho assumimos que a estrutura de medição necessária está implementada no interior da rede.

O índice de normalização é necessário para compatibilizar a dimensão das grandezas no problema (5), evitando que um dos termos tenha um peso maior e desproporcional em relação ao outro podendo gerar soluções tendenciosas.

Pode-se empregar a tecnologia MPLS (Multiprotocol Label Switching) para a marcação dos pacotes UDP para que eles sejam encaminhados pela rota de maior Surplus.

\section{B. Algoritmo de Controle de Admissão}

$\mathrm{O}$ algoritmo de controle de admissão é simples. Ele trabalha em conjunto com o algoritmo de roteamento apresentado na seção $A$ e também utiliza o mecanismo de preços dinâmico apresentado na equação (5).

Quando um fluxo UDP deseja ingressar na rede, primeiramente são verificados os caminhos válidos que tenham disponibilidade de recursos maior ou igual a $B_{\text {min }}$. Caso não exista o fluxo é descartado, já que seu ingresso na rede traria apenas prejuízo aos fluxos existentes porque sua utilidade seria nula $\left(U_{u d p}=0\right)$. Caso existam caminhos que atendam $x>B_{\text {min }}$, é calculada a rota que maximize o Surplus.

Por último, para determinar quanto de banda será reservado para o fluxo, é verificado o custo da rota escolhida e quanto o fluxo UDP poderá pagar (cofre: quantidade de recursos financeiros que o usuáio dispõe para uso no serviço de transmissão de dados). Caso o fluxo UDP não tenha fundos (cofre) para uma reserva maior ou igual ao seu $B_{\min }$ ele será descartado. Caso contrário, ele irá ingressar na rede com uma reserva proporcional a capacidade de pagar pela desutilidade causada aos fluxos TCP.

Funcionamento do mecanismo de controle de admissão:
1: O caminho válido que maximiza o surplus tem disponibilidade para reserva de banda $X$, onde $X>B_{\min }$ (obs: a reserva primeiramente é limitada pela capacidade física disponível no enlace, só após esta primeira verificação que o controle por preço dinâmico é acionado).

2: Verifica-se quanto o fluxo está disposto a pagar:

2.1: Não tem fundos (cofre $<B_{\min } \sum_{i=1}^{n} P_{i}$ ) para pagar por uma reserva maior que $B_{\text {min }}$;

Então: O fluxo é descartado;

2.2: Tem fundos (cofre $\geq B_{\min } \sum_{i=1}^{n} P_{i}$ ) para pagar por uma reserva referente a um valor entre $B_{\min } e B_{\max }$; Então: O fluxo é admitido com reserva de banda igual ao proporcional de sua capacidade de fundos, sempre respeitando a capacidade de banda disponível no link $\left(B_{\min } \leq x \leq B_{\max }\right)$;

2.3: Tem fundos para pagar por uma reserva maior que $B_{\max }$

Então: O fluxo é admitido com reserva de banda igual a $B_{\max }$ para $x \geq B_{\max }$. Assim, evita-se despedício de recursos financeiros e de rede, já que uma reserva maior que $B_{\max }$ não trará benefícios adicionais ao fluxo, visto que sua utilidade já é máxima $\left(U_{u d p}=1\right)$ e uma reserva maior iria onerar desnecessariamente o usuário.

\section{Simulação e Resultados}

Em nossa simulação, optamos por criar um simulador desenvolvido em linguagem $\mathrm{C}$, devido à elevada complexidade de implementação dos algoritmos de controle de admissão e roteamento proposto e dos cenários investigados em simuladores de rede de propósitos gerais. Estudamos três políticas de roteamento e controle de admissão para fins de análise de desempenho das propostas.

\section{A. Políticas de Roteamento e Admissão}

- (STDN): Esta forma de roteamento e política de admissão simula o comportamento atual da internet, onde todos os fluxos tendem a utilizar a rota de maior capacidade, como o implementado pelo OSPF [9]. O controle de admissão dos fluxos UDP é definido pela capacidade do enlace, isto é, o enlace aceita uma nova reserva de um fluxo UDP até que sua capacidade esteja esgotada com reservas UDP.

- (CAC): Esta forma implementa o algoritmo de controle de admissão para os fluxos UDP, conforme a subseção IV.B. Todos os fluxos TCP e UDP são roteados normalmente, ou seja, tendem a utilizar a rota de maior capacidade.

- (Surplus+CAC): Esta forma implementa o algoritmo de roteamento surplus e o algoritmo de controle de admissão para os fluxos UDP, conforme apresentado na seção IV. Os fluxos TCP são roteados pelo procedimento usual.

Para todas as formas, assumimos que os fluxos TCP compartilham os recursos disponíveis de uma forma justa implementada através do algoritmo "Max-Min Fair" [10]. 
Consideramos para a segunda e para a terceira forma que os fluxos UDP podem ser classificados de acordo com seu cofre, como Pobre ou Rico. Conforme já mencionado, cofre é um parâmetro que indica quanto um fluxo (aplicação/usuário) pode pagar para utilizar a rede, isto é, quanto ele está disposto a pagar pelo prejuízo (desutilidade) que irá ser gerado aos fluxos TCP com a sua entrada. Em nossas simulações consideramos que $80 \%$ dos fluxos UDP são pobres e o restante ricos. Este critério é utilizado para simular os tipos de serviços diferenciados que podem ser oferecidos aos diferentes tipos de usuário de acordo com cada provedor de serviço.

\section{B. Parâmetros de Entrada}

Utilizamos na simulação os seguintes parâmetros. Chegadas de novas conexões modeladas por um processo de Poisson de taxa 3,5 (chegadas/segundo). Para os fluxos UDP, o tempo de permanência no sistema foi modelado por meio de uma distribuição exponencial. $B_{\max }$ e $B_{\min }$ foram respectivamente de $2,5 \mathrm{Mb}$ e $0,05 \mathrm{Mb}$. Os cofres para fluxos UDP Ricos foram representados por números aleatórios entre 10 e 5 u.m. (unidades monetárias) e para fluxos Pobres entre 5 e 1 u.m. Para os fluxos TCP, consideramos uma transmissão de arquivos de tamanho que varia de $1 \mathrm{Mb}$ até $15 \mathrm{Mb}$ aleatóriamente e $B_{\text {min }}$ de $5 \mathrm{~Kb}$. Um fluxo TCP realiza até 3 tentativas de se obter banda maior ou igual a $B_{\min }$ antes do fluxo TCP desistir, o que representa a insatisfação do usuário e o abandono da rede. A constante monetária usada na equação do preço (5) foi igual a 1 .

\section{Resultados da Simulação}

As métricas avaliadas para testar a eficiência de nossa proposta, foram: utilidade média da rede para os fluxos TCP e UDP, o percentual de bloqueios dos fluxos UDP, bloqueios por recursos insuficientes $\left(x<B_{\min }\right)$, cofre insuficiente para fluxos UDP, percentual dos fluxos UDP e TCP atendidos, percentual dos fluxos TCP desistentes.

Utilizamos uma topologia inspirada na rede IPÊ [2] como topologia de testes. Para todos os resultados obtidos nesta topologia, foi computado o intervalo de confiança de $1 \%$ com grau de confiança de $99,7 \%$.

Nas tabelas a seguir apresentamos um sumário dos parâmetros de desempenho obtidos para três diferenes cenários: $i)$ 90\% de fluxos TCP e $10 \%$ de fluxos UDP no sistema, $i i) 75 \%$ TCP e $25 \%$ UDP, e $i i i) 50 \%$ TCP e $50 \%$ UDP. Na simulação foram geradas 28.063 chegadas para o caso $(i), 27.837$ chegadas para $(i i)$ e 28.216 chegadas para (iii).

As Tabelas I, II e III mostram os valores das utilidades médias obtidas para os fluxos TCP e UDP nos três esquemas simulados. Pode-se observar que os algoritmos propostos quando comparados com a abordagem tradicional (STDN) proporcionaram uma sensível melhora no desempenho dos fluxos TCP, sem causar um impacto significativo na utilidade dos fluxos UDP. Conforme nossa expectativa, o esquema que combina o roteamento com o controle de admissão UDP (Surplus+CAC) apresentou melhores resultados do que o esquema operando apenas com o controle de admissão CAC.
Isso se deve ao fato do roteamento Surplus selecionar caminhos alternativos que em geral estão menos congestionados.

A pequena vantagem do esquema tradicional STDN no que diz respeito ao desempenho do tráfego UDP (utilidade média) deve ser melhor analisada. Primeiramente, as utilidade médias foram computadas apenas para os fluxos que efetivamente usaram o sistema, ou seja, os fluxos que foram admitidos.

As tabelas IV, V e VI mostram outros parâmetros importantes para a análise do desempenho. A quantidade de fluxos UDP bloqueados (por falta de recursos disponíveis) e fluxos TCP desistentes é maior no esquema tradicional STDN em todos os cenários simulados. Nesse caso, quando os primeiros fluxos UDP entram na rede, estes realizam a reserva sem nenhum mecanismo que os regule, ou seja consumindo o máximo de recursos $\left(B_{\max }\right)$ e experimentando utilidades elevadas. Novos fluxos que chegam não conseguem garantir o mínimo de recursos $B_{\min }$ para que a rede forneça alguma utilidade para as aplicações/usuários, refletindo no maior número de bloqueios UDP.

\section{TABELA I}

UTILIDADES MÉDIAS DOS FLUXOS TCP E UDP PARA A REDE COM $90 \%$ DE TCP E $10 \%$ DE UDP

\begin{tabular}{|c|c|c|}
\hline & $U_{t c p}$ & $U_{u d p}$ \\
\hline \hline Surplus+CAC & 0,53 & 0,47 \\
\hline STDN & 0,31 & 0,49 \\
\hline CAC & 0,46 & 0,44 \\
\hline
\end{tabular}

TABELA II

UTILIDADES MÉDIAS DOS FLUXOS TCP E UDP PARA $75 \%$ DE TCP E $25 \%$ DE UDP

\begin{tabular}{|c|c|c|}
\hline & $U_{t c p}$ & $U_{u d p}$ \\
\hline \hline Surplus+CAC & 0,50 & 0,45 \\
\hline STDN & 0,29 & 0,47 \\
\hline CAC & 0,44 & 0,42 \\
\hline
\end{tabular}

TABELA III

UTILIDADES MÉDIAS DOS FLUXOS TCP E UDP PARA $50 \%$ DE TCP E $50 \%$ DE UDP

\begin{tabular}{|c|c|c|}
\hline & $U_{t c p}$ & $U_{u d p}$ \\
\hline \hline Surplus+CAC & 0,39 & 0,38 \\
\hline STDN & 0,25 & 0,42 \\
\hline CAC & 0,36 & 0,37 \\
\hline
\end{tabular}

Por outro lado, os esquemas Surplus+CAC e CAC por admitirem mais fluxos têm que dividir os recursos de rede entre um número maior de usuários, o que também pode ter contribuído pela ligeira redução nas utilidades médias UDP. O algoritmo de controle de admissão proposto, além de garantir menor número de abandono e maiores utilidades para os fluxos TCP funciona como regulador do tamanho das reservas realizadas pelos fluxos UDP.

Os cenários onde os fluxos UDP foram roteados pelo algoritmo Surplus, os direcionam por caminhos menos congestionados, onde a reserva tende a ser maior e o impacto 
TABELA IV

DESEMPENHO DOS FLUXOS UDP E TCP NO CRITÉRIO STDN

\begin{tabular}{|l|c|c|c|c|}
\hline & Atendimentos & Bloqueios & Atendimentos & Abandono \\
& & & & \\
& UDP(\%) & UDP $(\%)$ & TCP $(\%)$ & TCP(\%) \\
\hline $\mathbf{9 0 \% - 1 0 \%}$ & 45,97 & 36,44 & 26,60 & 42,40 \\
\hline $\mathbf{7 5 \% - 2 5 \%}$ & 39,96 & 51,22 & 20,18 & 53,34 \\
\hline $\mathbf{5 0 \% - 5 0 \%}$ & 34,23 & 58,91 & 19,17 & 59,40 \\
\hline
\end{tabular}

TABELA V

DESEMPENHO DOS FLUXOS UDP E TCP NO CRITÉRIO CAC

\begin{tabular}{|l|c|c|c|c|}
\hline & Atendimentos & Bloqueios & Atendimentos & Abandono \\
& & & & \\
& UDP(\%) & UDP(\%) & TCP(\%) & TCP(\%) \\
\hline $\mathbf{9 0 \% - 1 0 \%}$ & 47,44 & 36,80 & 38,13 & 35,71 \\
\hline $\mathbf{7 5 \% - 2 5 \%}$ & 40,61 & 49,54 & 37,70 & 40,17 \\
\hline $\mathbf{5 0 \% - 5 0 \%}$ & 38,83 & 51,21 & 34,85 & 43,86 \\
\hline
\end{tabular}

TABELA VI

DESEMPENHO DOS FLUXOS UDP E TCP NO CRITÉRIO SURPLUS+CAC

\begin{tabular}{|l|c|c|c|c|}
\hline & Atendimentos & Bloqueios & Atendimentos & Abandono \\
& & & & \\
& UDP $(\%)$ & UDP $(\%)$ & TCP $(\%)$ & $\operatorname{TCP}(\%)$ \\
\hline $\mathbf{9 0 \% - 1 0 \%}$ & 47,96 & 33,83 & 43,37 & 28,08 \\
\hline $\mathbf{7 5 \% - 2 5 \%}$ & 44,32 & 39,84 & 40,03 & 35,17 \\
\hline $\mathbf{5 0 \% - 5 0 \%}$ & 42,41 & 41,30 & 39,01 & 38,33 \\
\hline
\end{tabular}

sofrido pelos fluxos TCP é minimizado. Este critério foi o que apresentou os melhores índices prevenindo que os fluxos UDP prejudicassem mais diretamente os fluxos TCP.

Assim, pelos resultados de simulação apresentados para os três diferentes cenários a nossa proposta foi capaz de: melhorar o desempenho dos fluxos TCP evitando o estrangulamento, admitir uma maior quantidade de fluxos UDP, reduzir as desistências dos fluxos TCP. Além disso, a proposta implementa um mecanismo dinâmico de tarifação que promove o uso mais eficiente dos recursos da rede e que pode ser configurado (p.ex. através da constante monetária $C$ ) pelos provedores de telecomunicações para aumentar o retorno financeiro com a venda dos serviços.

\section{CONClus Ão}

Apesar de existirem algumas propostas de mecanismos de controle de congestionamento dos fluxos UDP como o trabalho [11], em nosso artigo apresentamos uma proposta inovadora para a regulação do tráfego UDP de acordo com o impacto causado no tráfego TCP. Utilizamos conceitos da teoria microecomômica para formular os procedimentos e permitir o desenvolvimento de mecanismos de regulação baseados em tarifação. Realizamos ainda a proposta de um algoritmo de controle de admissão e um algoritmo de roteamento para fluxos UDP. Resultados de simulação mostraram que nossas propostas são consistentes e garantem um melhor desempenho para o sistema.

Alguns pontos merecem ainda um maior trabalho de investigação em pesquisas futuras. A estrutura de medição para inferir o desempenho dos fluxos TCP no interior da rede (determinação dos $D_{k}$ ) precisa ser melhor detalhada. Experimentos de simulação para uma grande variedade de parâmetros de entrada também são necessários para uma melhor avaliação do potencial da proposta em ambientes reais. Estudos sobre o comportamento dos usuários sob sistemas de tarifação dinâmicos também podem se importantes antes da implementação prática dos mecanismos nos provedores de serviço de redes.

Existe também o interesse em se investigar funções utilidade para outros parâmetros de desempenho, tais como atraso de pacotes, perda de pacotes, etc. Funções em mais de uma dimensão, por exemplo combinando banda com atraso, também poderiam ser estudadas apesar do aumento na complexidade do sistema. Uma outra possibilidade é desenvolver uma interface para a configuração das Políticas de desempenho.

Por fim, acreditamos que sistemas de tarifação diferenciados e dinâmicos constituem importantes mecanismos não só visando o retorno financeiro imediato para os provedores de serviço, mas principalmente proporcionando regulação do tráfego na rede e incentivos para o controle de congestionamento e suporte a qualidade de serviço.

\section{AGRADECIMENTOS}

Os autores gostariam de agradecer todo o apoio recebido pelo Instituto Militar de Engenharia (IME) e pela Coordenação de Aperfeiçoamento de Pessoal de Nível Superior (CAPES) sem os quais este trabalho não seria possível.

\section{REFERÊNCIAS}

[1] Internet World Stats, Estatística de uso da Internet. http://www.internetwordstats.com , Online; acessado 12-03-2007.

[2] RNP - Rede Nacional de Ensino e Pesquisa, "Topologia-Rede IPÊ". http://www.rnp.br/backbone/index.php, Online; acessado 20-Novembro2006.

[3] Hal R. Varian, "Intermediate microeconomiacs: A Modern Approach", WW Norton \& Co. Ltd, 2006.

[4] S. Shenker, "Fundamental design issues for the future internet," IEEE Journal on Selected Areas in Communication, v. 13, Setembro 1998.

[5] S.Floyd and K. Fall, "Promoting the Use of End-to-End Congestion Control in the Internet" IEEE/ACM Transactions on Networking, 1998.

[6] M. Mathis and J. Semke and J. Mahdavi and T. Ott, "The macroscopic behavior of the TCP congestion avoidance algorithm," Computer Communication, v. 27, p. 67-82, 1997.

[7] R. Liao and A. T. Campbell, "Dynamic edge provisioning for core IP networks," IEEE/IWQoS - Workshop on Quality of Service , p. 148157,Junho 2000.

[8] P. Key and L. Massoulié and A. Bain and F. Kelly, "Fair Internet traffic integration:network flow models and analysis," Annales des Telecommunications, N. 59, p. 1338-1352, 2004.

[9] T. M. Thomas, "OSPF Network Design Solutions," Cisco Press, 1998.

[10] Jean Yves Le Boudec, "Rate Adaption, Congestion Control and fairness: A Tutorial," EPFL - Ecole Polytechnique Fédérale de Lausanne,Dezembro 2000.

[11] Eddie Kohler and Mark Handley and Sally Floyd, "Designing DCCP: Congestion Control Without Reliabity," SIGCOMM,p.11-15, Setembro 2006. 